\title{
Coherence-based SAR tomography for spaceborne applications
}

\author{
Matteo Nannini*, Michele Martone, Paola Rizzoli, Pau Prats-Iraola, \\ Marc Rodriguez-Cassola, Andreas Reigber, Alberto Moreira \\ Microwaves and Radar Institute, German Aerospace Center (DLR), D-82234 Oberpfaffenhofen, Germany
}

\section{Abstract}

Future SAR missions will provide three-dimensional images of semi-transparent media, such as vegetation and ice, through SAR tomography. Access to information on the internal structure of these volume scatterers is a key factor for a better understanding of ecosystem dynamics and climate change. Because of this, several concepts are nowadays examined to implement SAR tomography in a spaceborne framework.

In order to do that, it is necessary to gather different observations of the area of interest. Unfortunately, a consequence of the time that elapses between acquisitions is that the electromagnetic properties of the medium may vary. This implies that, there may be inconsistencies in the acquired data, leading to errors in the final inversion. A solution to partially cope with this temporal decorrelation, is to acquire data employing two or more sensors operating with a reduced (or even absent) temporal gap and then to collect several acquisitions at different time instants. By means of this imaging concept, the required line-of-sight diversity is granted and the desired resolution in the height direction ensured. In this way, sets of temporal decorrelation-free interferometric coherences can be built and the vertical scattering profile can be retrieved via coherence-based tomography.

This contribution analyses a two-sensor system like TanDEM-X [Krie 07], Tandem-L [More 15], or SAOCOM-CS [Davi 14]. In particular, the potential of coherence-based tomography are shown with data acquired with the TanDEM-X sensors for boreal and Amazon forest. In addition, a technique to partially cope with temporal decorrelation through covariance matrix filtering is also presented in the paper.

Keywords: Synthetic Aperture Radar (SAR), SAR Tomography, Companion Satellites, Bi-static SAR, Volume Scatterers, Vegetation.

\section{Introduction}

The next generation of spaceborne missions will operationally employ SAR tomography to achieve real three-dimensional imaging of volume scatterers. This type of semi-transparent media consists of multiple particles, distributed over the height, sharing the same resolution cell in the final SAR image. SAR tomog-

*Principal Corresponding Author 
raphy can resolve those contributions since it achieves a vertical resolution through the construction of a synthetic aperture (tomographic aperture) in the perpendicular line-of-sight (PLOS) direction. It is usually performed after standard 2D SAR processing and operates on a stack of coregistered images.

Several applications can profit from the information retrieval obtained by tomography. In particular, monitoring of bio-, cry- and hydrosphere will become much more efficient and easier, granting access to information regarding the internal structure of such volume scatterers [More 15]. This will improve the comprehension of the environmental Earth's dynamics in a global manner. In this context, for example, a mission like TanDEM-L, among its several goals aims to measure and monitor the three-dimensional forest structure and biomass to better understand the carbon cycle, to determine the three-dimensional ice structure and evaluate the melting processing to improve predictions of future sea level rise. Especially for vegetation, gathering information about its internal structure is of fundamental importance in determining the corresponding biomass [Bohn 17].

One of the main challenge to face, when implementing tomography, is the trade-off between number of acquisitions and desired resolution/ambiguity rejection performance [Nann 09]. Factors that are directly related to the sampling of the tomographic aperture. Hence, various surveys of the same area of interest have to take place in order to collect sufficient data to synthesize the desired aperture and enable the vertical resolution capability of SAR tomography.

Moreover, the problem in acquiring more passes in a spaceborne framework is that same scattering and weather conditions are often not granted since, usually, several days elapse between two acquisitions over the same area. Because of that, inconsistencies in the scene due to temporal changes, make it difficult to achieve an accurate information retrieval. Ideally, one would simultaneously acquire a set of passes with defined baselines. This usually happens in airborne campaigns where, within a few hours, several tracks can be gathered [Nann 12] [Teba 16]. This would allow classical single-look complex (SLC) backprojection tomographic approaches to be applied [Reig 00]. However, at the present time, a spaceborne implementation of such constellations has strong feasibility constraints. To partially solve this problem, one can refer to the Van Cittert-Zernike theorem and perform tomographic imaging via coherent mapping [Krie 09]. The basic idea behind this approach is that, if a set of single-pass interferometric complex coherences is available, each of those will exhibit a negligible decorrelation due to temporal changes. This is because, the data building the coherences are acquired approximately at the same time instant. These coherences can then be combined to retrieve the vertical distribution of scatterers from the associated volume decorrelation effect. Related studies are currently underway to design spaceborne SAR missions that involve multiple sensors able to acquire quasi-simultaneous data. The approach described can, however, already be demonstrated thanks to the TanDEM-X (TerraSAR-X Add-oN for Digital Elevation Measurements) mission, in which the two radar 
systems TSX (TerraSAR-X) and TDX (TanDEM-X) constitute the first single-pass SAR interferometer in space. Its main objective has been the generation of a global digital elevation model with unprecedented precision: $12 \mathrm{~m}$ resolution, $10 \mathrm{~m}$ absolute height accuracy and $2 \mathrm{~m}$ relative height accuracy. The possibility to properly analyse the performance of coherence-based tomography came with the beginning of the TanDEM-X science phase in October 2014, when the two satellites were being moved apart. In particular, the analyses presented in later sections are based on data acquired in the so-called pursuit monostatic mode, in which the two satellites independently acquire two images with a temporal separation of about ten seconds. The technique has been tested over boreal as well as over tropical forest. Preliminary results of coherence-based tomography have been shown in [Pard 13]. At the time of previous studies, however, TanDEM-X science phase data were not yet available and, as a consequence, the potential of the technique could only partially be shown due to the poor tomographic resolution.

The present study is also intended as a proof-of-concept for future missions such as Tandem-L, where the L-band center frequency implies that media such as vegetation or ice appear as semi-transparent volume scatterers [Reig 00; Nann 12; Teba 16].

It is understood that the set of multi- and single-pass interferograms will include changes in the area of interest. The experimental results, however, suggest that the sensitivity to such changes is much reduced when tomographic imaging is based on single-pass, rather than repeat-pass, interferometry. The objective of this paper is therefore twofold: firstly, it is to experimentally confirm the superiority, in practice, of coherencebased tomography over classical SLC backprojection approaches in the context of spaceborne SAR. Secondly, the objective is to demonstrate the potential of the proposed technique for vegetation analysis.

In addition the present study introduces a matrix filtering technique for improving the robustness of super-resolution, covariance-based methods to decorrelation due to temporal changes in repeat- and singlepass data.

\section{Theoretical Background}

Coherence-based tomography can be applied to estimate the vertical distribution of scatterers on the basis of interferometric complex coherences acquired with negligible or even zero temporal decorrelation. In the spaceborne case, this type of data is provided by missions such as TanDEM-X and the future L-band Tandem-L mission. In [Zebk 92], the complex coherence is modeled as the product of independent decorrelation factors. For example, the complex coherence $\gamma$ can be expressed as:

$$
\gamma=\gamma_{\mathrm{vol}} \cdot \gamma_{\mathrm{temp}} \cdots
$$


where $\gamma_{\mathrm{vol}}$ and $\gamma_{\text {temp }}$ stand for the volumetric and temporal decorrelation, respectively. The key insight behind coherence-based tomography is that the measured volume decorrelation is the normalized Fourier transform of the vertical distribution profile $p(h)$ :

$$
\gamma_{\mathrm{vol}}=\frac{\int_{0}^{H} p(h) \exp \left(j k_{z} h\right) d h}{\int_{0}^{H} p(h) d h}
$$

where $k_{z}$ represents the vertical wavenumber.

The retrieval of the normalized vertical distribution of scatterers can be achieved by exploiting the set of complex coherences with methods such as those proposed in [Clou 06; Teba 08]. The most direct way to obtain the desired information, however, is to adopt backprojection approaches as described in [Reig 00]. In doing so, it should be noted that coherence-based tomography then entails the backprojection of the complex coherences rather than a set of SLC images. The equivalence of the estimated vertical profile by the two types of backprojection has been demonstrated in [Teba 17]. Alternatively, classical spectral estimators such as Capon [Gini 02] can be applied to the covariance matrix derived directly from the SLCs without requiring interpolation. For the sake of comparison, the present study covers both both coherence backprojection and classical SLC-based approaches.

The covariance matrix resulting from a combination of repeat- and single-pass acquisitions will generally present a block-diagonal structure, since the coherence is typically much higher for pairs of acquisitions with a small temporal baseline. This characteristic can be exploited by filtering the covariance matrix to improve the robustness with respect to temporal decorrelation as discussed in Section 4 .

\section{TanDEM-X Experimental Results}

The present evaluation of coherence-based tomography is based on data acquired during the pursuitmonostatic science phase of the TanDEM-X mission. In this phase of the mission, which begun in October 2014 and lasted for 5 months, the two satellites operated independently [Anno 15; Zink 14]. These data provide the line-of-sight diversity required for tomographic imaging, as the across-track baseline ranged between $0 \mathrm{~m}$ and $750 \mathrm{~m}$. The $76 \mathrm{~km}$ along-track separation implies negligible radio frequency interference between the radar signals and corresponds to a temporal baseline of about ten seconds. To evaluate the performance of coherence-based SAR tomography in different vegetation scenarios, results are presented for both boreal forest and a site in the Amazon rain forest. The data were processed with the tomographic workflow of the experimental interferometric processor TAXI of the DLR Microwaves and Radar Institute [Nann 16; Prat 10].

In terms of calibration, it should be noted that tomography requires a common phase referencing for the 
coherences in a stack. For the present study, the required phase screens were estimated via a singular value decomposition as described in [Nann 17] and the references therein.

\subsection{Boreal Forest}

Boreal forests are mainly composed of evergreen conifers such as spruces, larches and pines and are to be found at latitudes between 50 and 60 degrees North. The analysis in this section is based on a set of 14 TanDEM-X monostatic acquisitions of a site near the village of Vindeln, Sweden, collected between October and December 2014. The perpendicular baseline distribution is illustrated in Figure 1, which shows the baselines with respect to the common master for SLC backprojection as well as the pair-wise baselines exploited by the coherence-based approach. The tomographic aperture, which is given by the maximum baseline in the perpendicular line-of-sight direction, is about $400 \mathrm{~m}$ for the SLC-based processing and $300 \mathrm{~m}$ for coherence-based processing. While the impact on the resolution is negligible, the denser sampling for the SLC case results in an improved ambiguity/sidelobe suppression. However, despite such conditions, it can be seen that coherence-based tomography can provide accurate results due to the intrinsic exploitation of the quasi-simultaneous data.
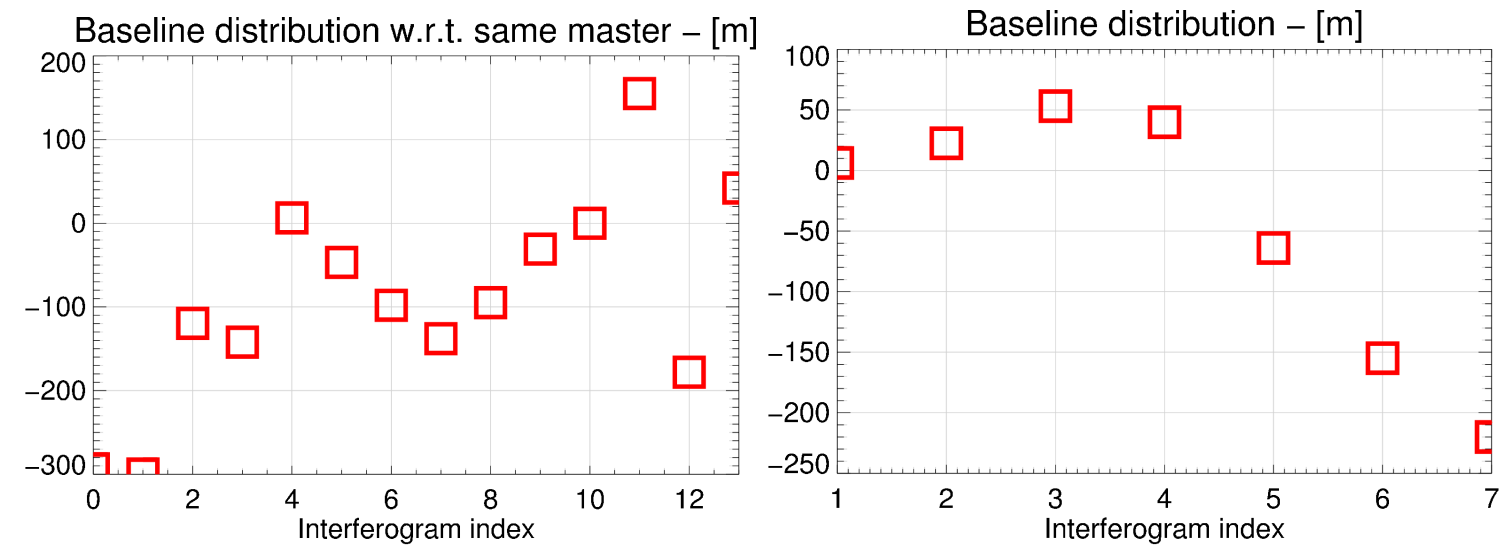

Figure 1: Baseline distributions for the acquisitions over boreal forest. Left: baselines of the single acquisitions with respect to a common master (track number 10). Right: the resulting pair-wise interferometric baselines.

Figure 2 presents a retrieval result, after normalization in azimuth, based on classical backprojection applied to the SLC data stack. The result is seen to be consistent with the TanDEM-X DEM. As expected, electromagnetic waves in X-band do penetrate into vegetation to some extent. In some areas, it is even possible to resolve the upper canopy and the signals backscattered on the ground. This may be due to the fact that there is, in those areas, a clear path through the forest in the radar line-of-sight. On the other hand, the energy is slightly spread around the expected phase center in the SLC backprojection result, rather than localized. This additional noise can be attributed to temporal decorrelation, since the phase center will not, in general, stay stable throughout the observation time corresponding to the full stack of SLCs. 

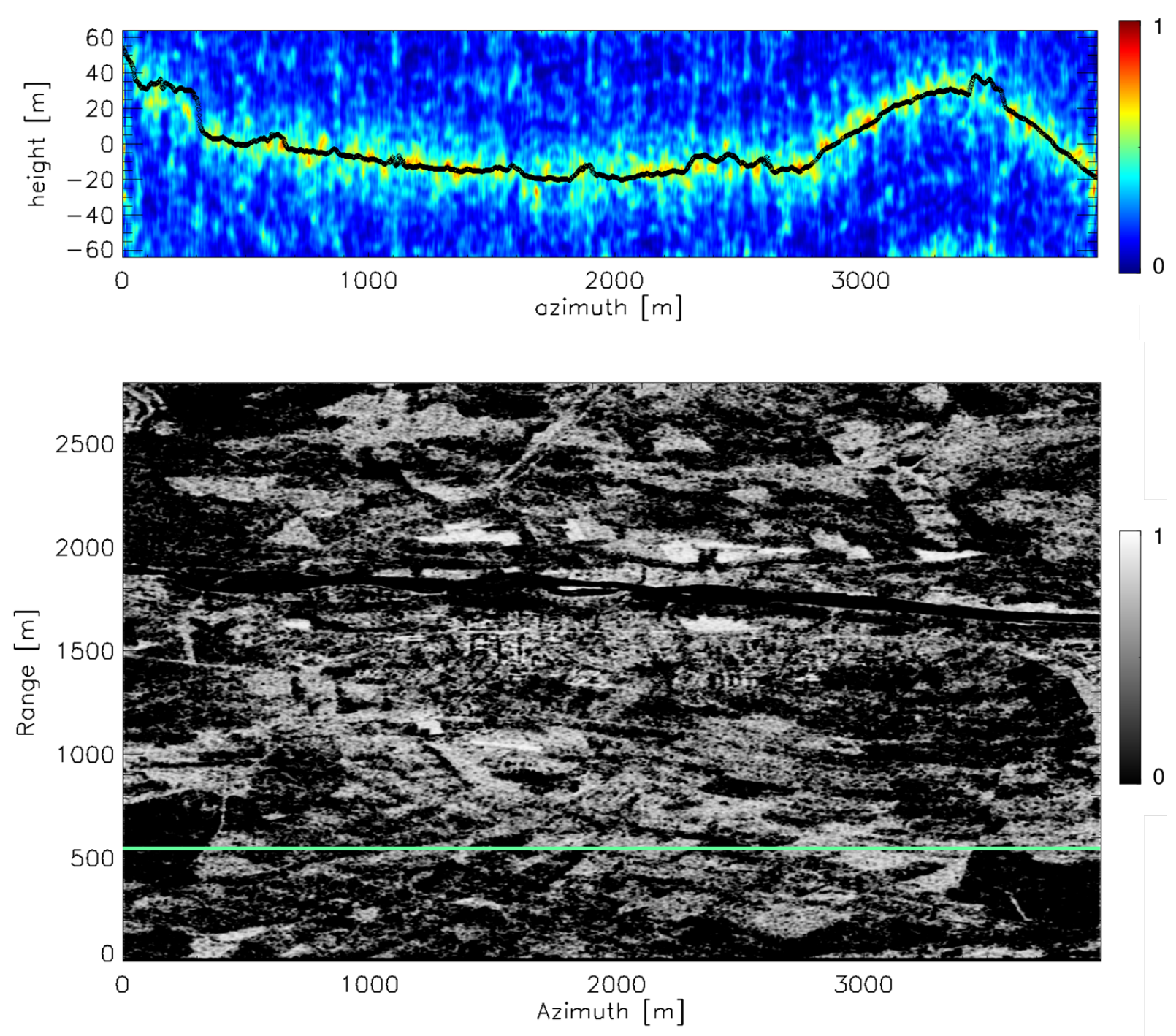

Figure 2: Results over boreal forest. Top: SLC backprojection tomogram with the TanDEM-X DEM overlaid in black. Bottom: Coherence image, in which dark areas indicate decorrelating scatterers like vegetation. The processed profile is indicated by the green line.

When the same data is considered in a pairwise manner, by building quasi single-pass interferometric coherences, coherence-based SAR tomography can be applied. This approach takes advantage of the fact that the scatterers' behavior remains mostly stable between the two observations. Figure 3 illustrates the results obtained.

Despite the sidelobes resulting from the non-optimal track distribution in terms of perpendicular baseline, coherence-based tomography leads to a more sharply focused profile. For instance, it is now possible to better identify the ground location and the canopy top. This result demonstrates that, given an appropriate constellation, coherence-based SAR tomography could allow the desired three-dimensional information to be retrieved, thereby partly addressing the issue of temporal decorrelation. 


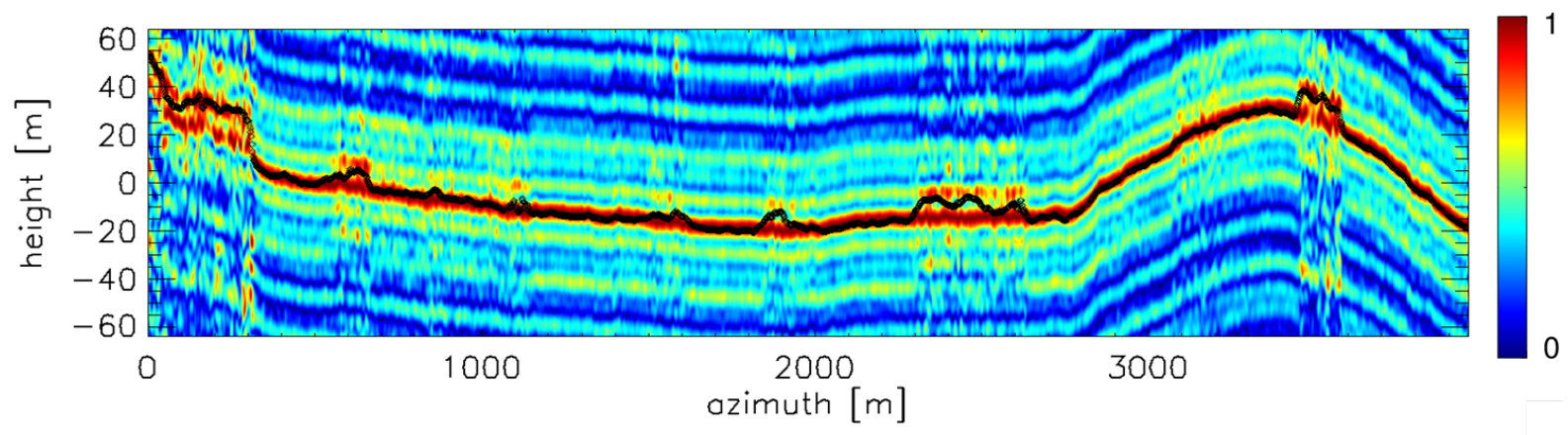

Figure 3: Results over boreal forest using coherence-based tomography with the TanDEM-X DEM overlaid in black. Besides the main contributions, the effects of the non-optimal sampling produce higher sidelobes within a range of $\pm 20 \mathrm{~m}$ around the main phase center.

Figure 4 shows the SLC-based Capon result. It can be seen that this retrieval is similar to the coherencebased one. It is worth mentioning that the Capon spectral estimator is based entirely on the inverse covariance matrix of the data. As the covariance matrix essentially consists of complex interferograms, the result obtained is almost exclusively determined by the information contained in the single-pass acquisitions.

A detailed analysis of the different methods in terms of imaging resolution is given in Section 6 .

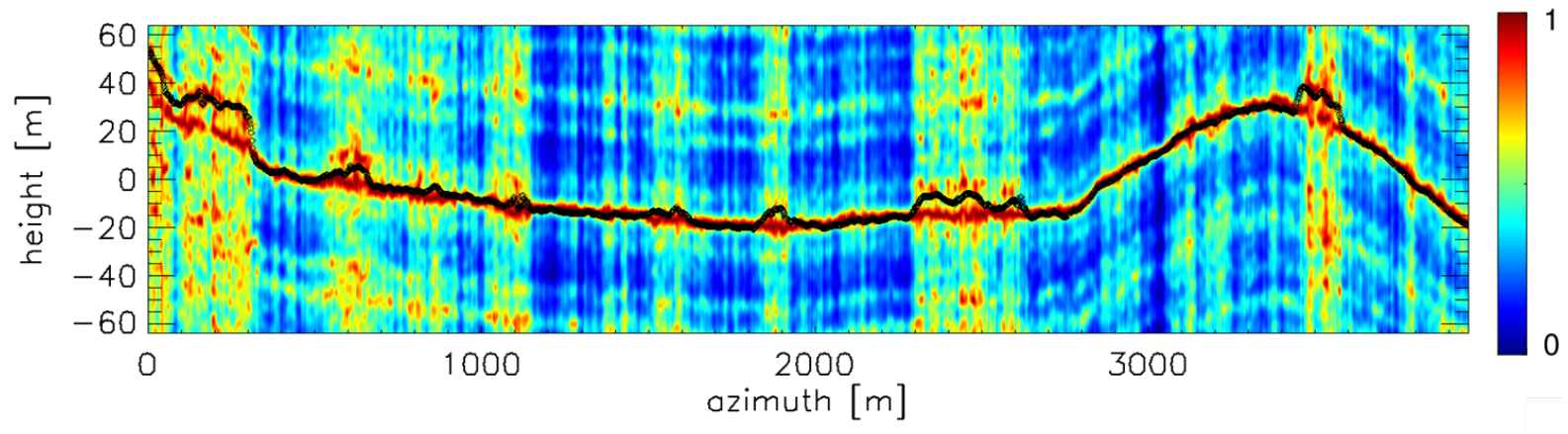

Figure 4: SLC-based Capon tomogram for boreal forest with the TanDEM-X DEM overlaid in black.

\subsection{Tropical Forest}

The world's tropical forests are located between \pm 23 degrees latitude and are highly heterogeneous in terms of tree species. The test site considered is located in the state of Rondônia, Brazil. The data set consists of 16 acquisitions carried out between October and December 2014. The perpendicular baselines for this configuration are reported in Figure 5. Compared to the interferometric baselines for the boreal data set, the baseline sampling is seen to be denser, which implies a larger height of ambiguity (i.e. the height variation corresponding to an interferometric phase change of $2 \pi$ ). One can observe that the interferometric baselines are less regular compared to those referred to a single master, implying higher sidelobes for the 
coherence-based inversion. Even so, the results will show that the coherence-based approach outperforms the standard SLC Fourier backprojection focusing in resolving the main vegetation layers. This conclusion is also supported by the discussion in Sections 5 and 6 , where the phase center location accuracy as well as the impulse response are investigated.
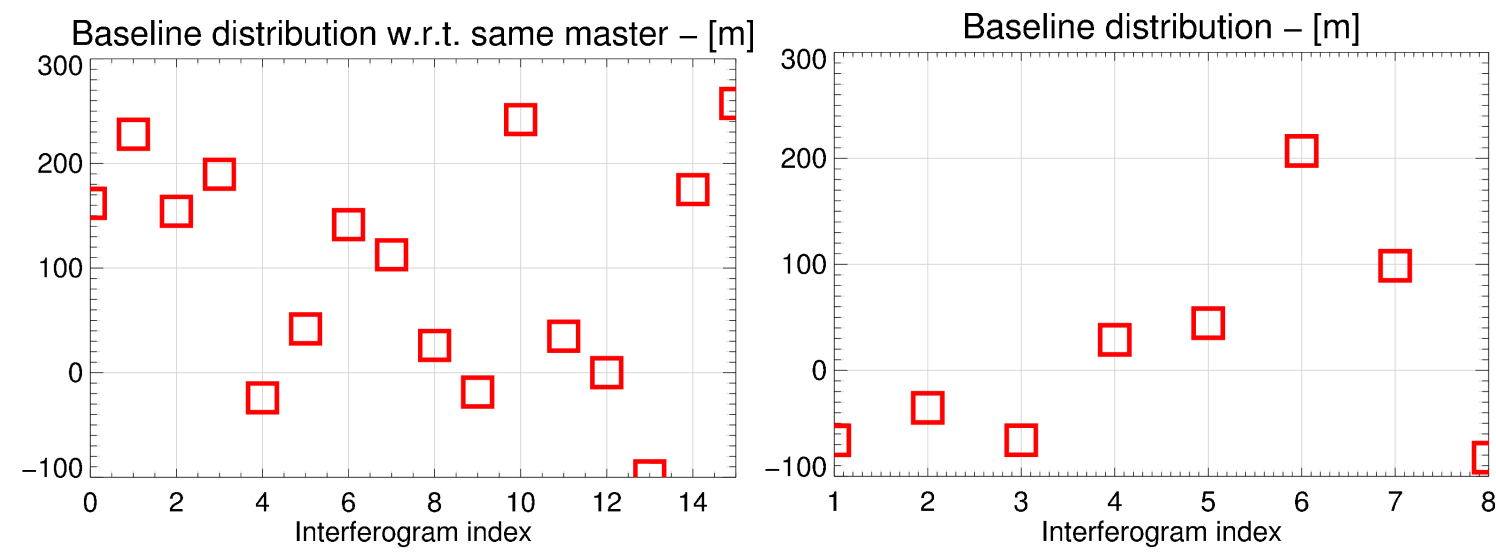

Figure 5: Baseline distributions for the acquisitions over tropical forest. Left: baselines of the single acquisitions with respect to a common master. Right: resulting pair-wise interferometric baselines.

As in the last section, Figures 6, 7, and 8 illustrate the results obtained for the tropical forest sight using classical SLC-based backprojection, coherence-based backprojection, and Capon beam forming, respectively. As before, the phase centers are well localized, which suggests a successful SVD-based calibration step. Moreover, the DEM consistency validates the retrieval for this scene, as the location of the ground phase center is unambiguous for the clear cuts it contains.

In the SLC backprojection result, the contributions from beneath the ground level are likely related to temporal decorrelation that arises due to phase center variations over the months-long observation period. In particular, variations and consequent decorrelation are to be expected due to changing weather conditions. In the boreal region, a lower precipitation rate is usually registered within a comparable time frame [NASA 00] and relatively stable backscattering properties are to be expected. For the tropics at latitudes around $10^{\circ}$, however, these months present the largest precipitation rate of the year [Onli 16]. Therefore, the corresponding data are more likely to be affected by temporal decorrelation than those collected for the boreal forest site. The detrimental effect on tomographic imaging is much less pronounced for the Capon estimator, since it profits from the averaging operation inherent in building the covariance matrix. The tomographic profile of the vegetation, ranging from azimuth coordinate $0 \mathrm{~m}$ to ca. $700 \mathrm{~m}$, is less stable than that observed in boreal areas. This is likely due to the heterogeneity of the underlying media as well as the seasonal weather conditions discussed previously. Since these kinds of forests are not sparse, there are no clear paths through the vegetation along the line-of-sight. In addition, the profiles indicate only a single 
dominant phase center in height due to the limited penetration of electromagnetic waves into vegetation in X-band.

Unfortunately, temporal decorrelation for volume scatterers is indeed highly problematic in the context of tomography [Sima 12]. In the interest of mitigating this effect as far as possible, the next section introduces a dedicated covariance matrix filtering approach.

\section{Covariance Matrix Filtering Approach}

As mentioned earlier in Section 2, the covariance matrix for a stack of sigle-pass interferometric measurements is expected to exhibit a block-wise structure. Temporal decorrelation primarily affects the elements of the covariance matrix that correspond to pairs of acquisitions that are not simultaneous. Therefore, one can prevent these repeat-pass matrix elements from degrading the final result by simply setting them to zero, as illustrated in Figure 9. The result of SLC-based Capon tomographic processing based on the thus filtered covariance matrix is shown in Figure 10.

The proposed filtering operation results in a tomogram with fewer outliers, particularly for vegetation, and a smaller response spread around the expected phase center. Moreover, the retrieval over the ground is more consistent with the DEM. The improvement in the reconstruction therefore suggests that the distortions in the initial result can be attributed to temporal changes. Moreover, testing the same procedure with data acquired over boreal forest, the improvement is not as noticeable as for the presented Amazon forest case. These results are consistent with the fact that temporal decorrelation is, for the seasons and test sites considered, more critical for tropical forests.

One can, however, also observe that removing some elements of the matrix potentially implies a slight degradation in the vertical resolution, since, depending on the constellation, some baselines may effectively be discarded entirely.

\section{Phase Center Accuracy: Comparison with TanDEM-X DEM}

To further validate the results, this section compares the TanDEM-X DEM to the location of the dominant phase center recovered. Since X-band typically penetrates very little into the vegetation canopy, with the exception of special cases such as sparse forests, the height of the tomographic peak response should generally correspond to the DEM surface. The test site selected for this analysis is the one in the Brazilian state of Rondônia, which contains clear cuts that also exhibit a single dominant phase center. Figure 11 shows the histogram of the difference between the TanDEM-X DEM and the heights derived from coherence- as well as Fourier SLC-based tomography. The results clearly show that the coherence-based tomographic DEM 

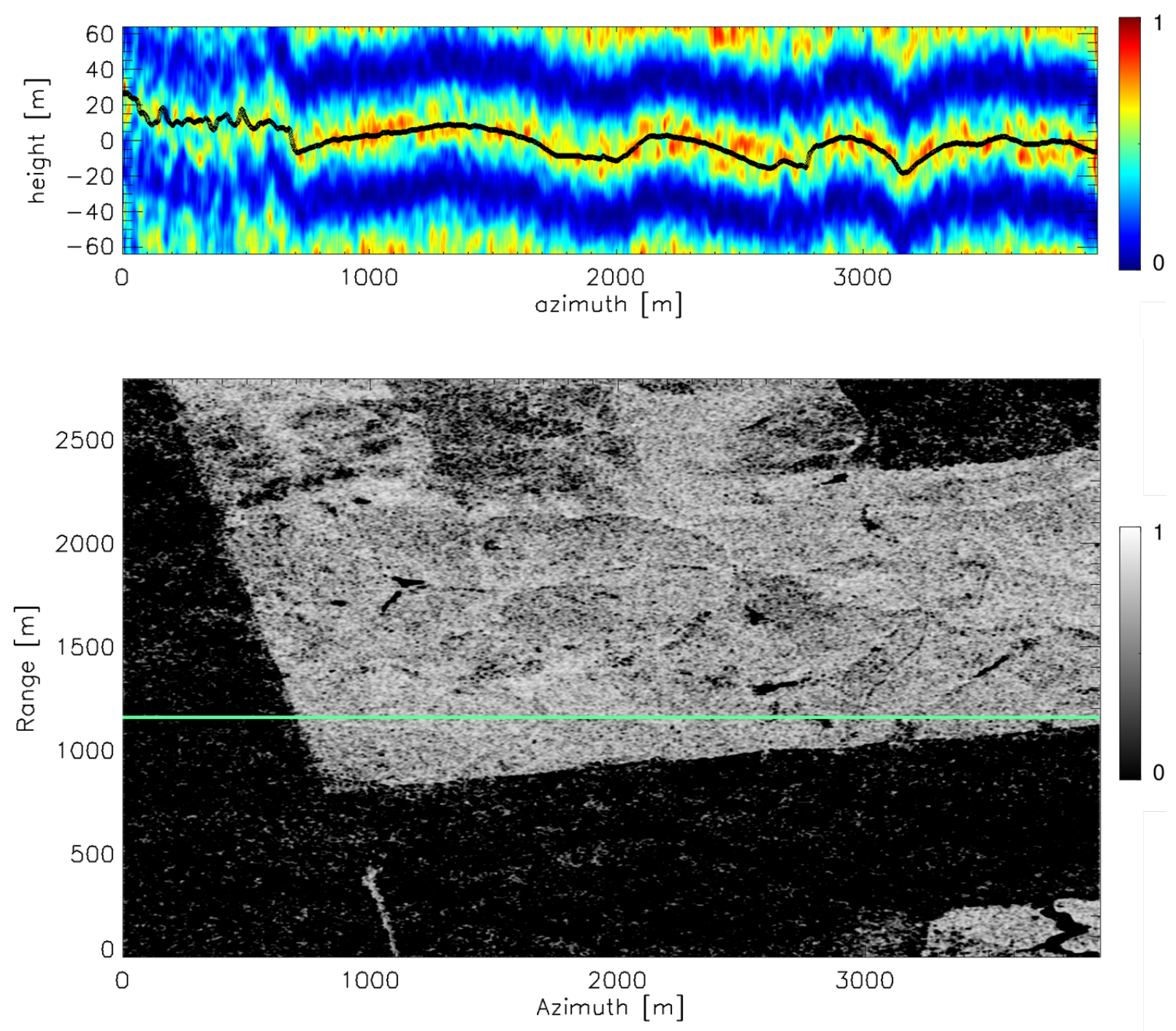

Figure 6: Results over tropical forest. Top: SLC backprojection tomogram with the TanDEM-X DEM overlaid in black. Bottom: Coherence image, in which dark areas indicate decorrelating scatterers such as vegetation. The processed profile is indicated by the green line. 




Figure 7: Results over tropical forest using coherence-based tomography with the TanDEM-X DEM overlaid in black.

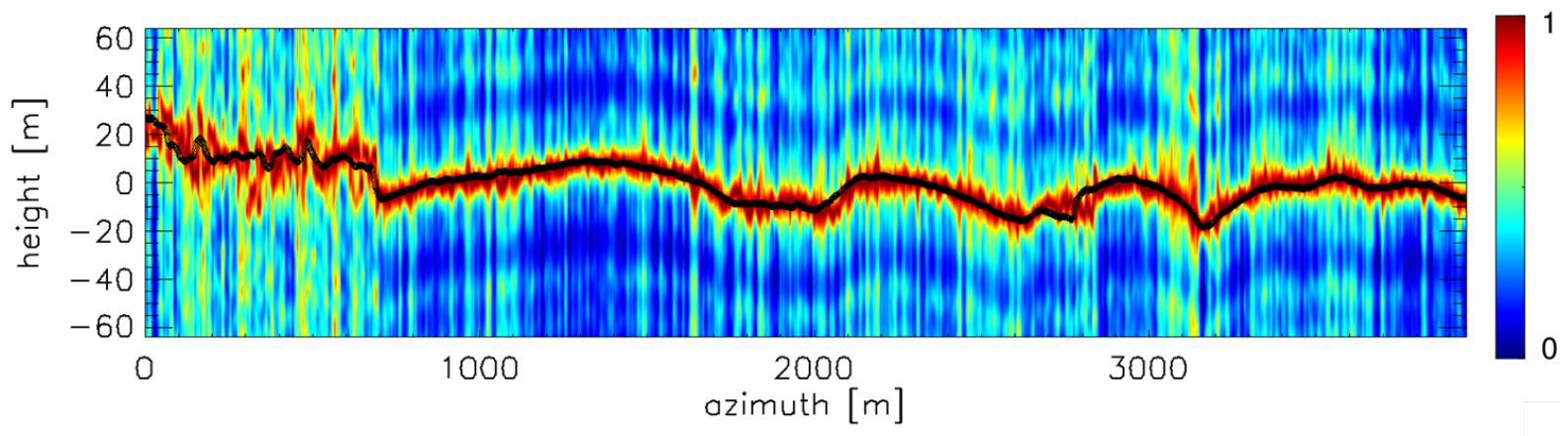

Figure 8: SLC-based Capon tomogram for tropical forest with the TanDEM-X DEM overlaid in black.

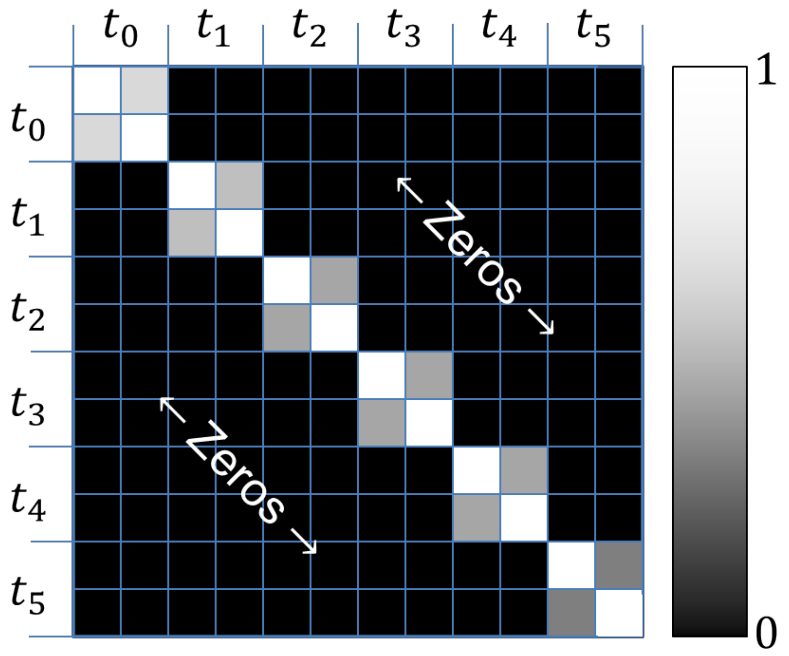

Figure 9: Filtering of the time sorted covariance matrix for multi- and single-pass acquisitions. In this example, ten acquisitions are gathered at the time instants $t_{0}, t_{1}, t_{2}, t_{3}, t_{4}$, and $t_{5}$. The black elements are explicitly set to zero, while the elements corresponding to quasi single-pass information are kept.

is much closer to the TanDEM-X one. This consistency also cross-validates the TanDEM-X DEM over vegetation. The two peaks at $\pm 60 \mathrm{~m}$ in the backprojection tomogram are due the ambiguities arising from the baseline distribution. 


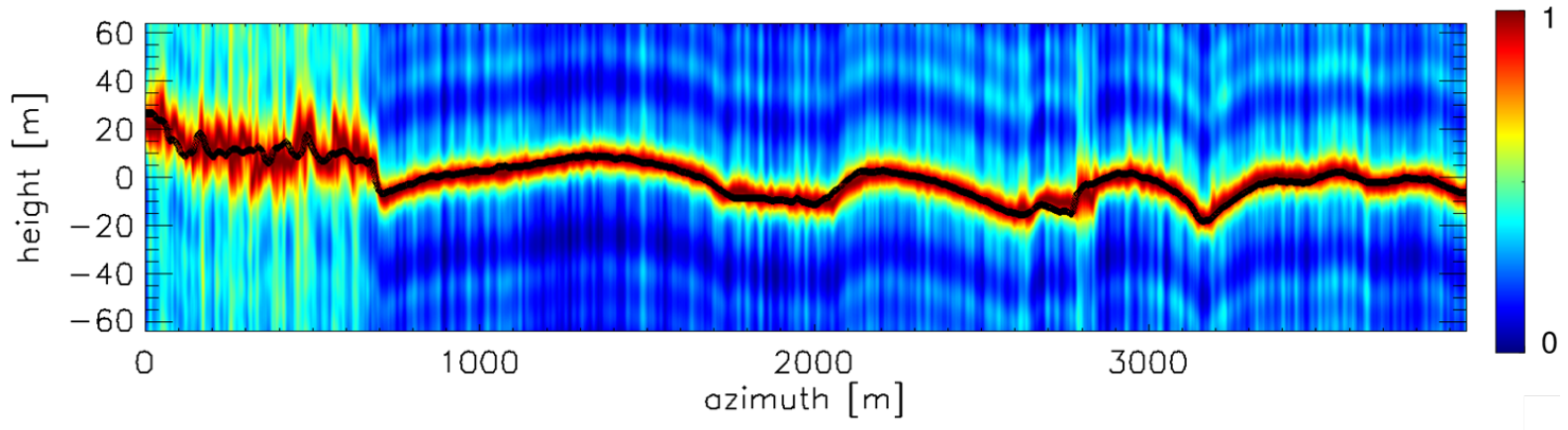

Figure 10: SLC-based Capon tomogram for tropical forest obtained on the basis of the filtered covariance matrix. The TanDEM-X DEM is overlaid in black.

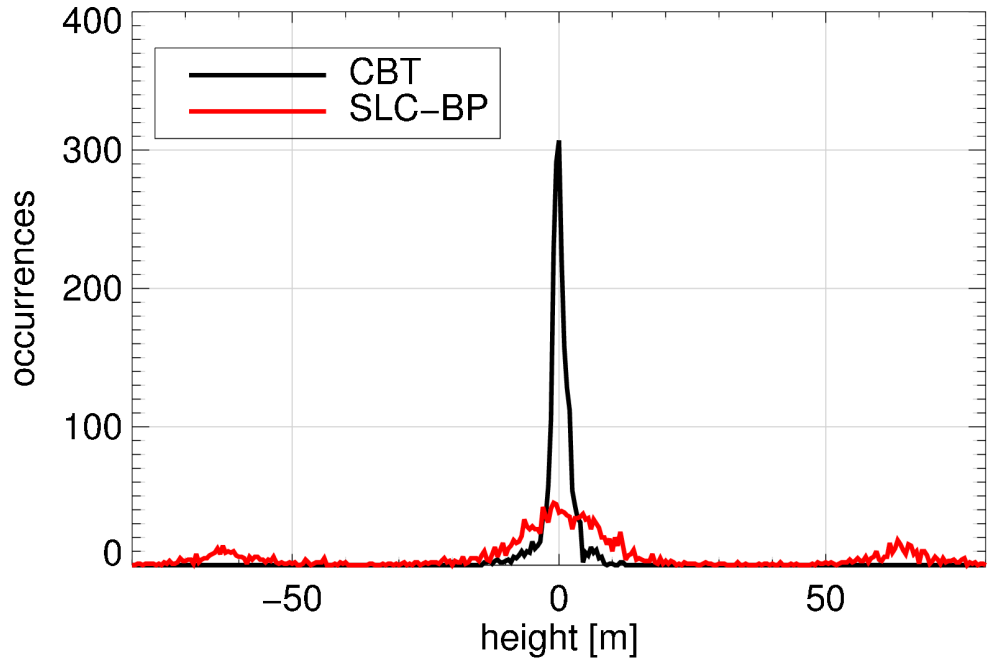

Figure 11: Histogram of the difference between the TanDEM-X DEM and the one retrieved from tomography using coherencebased tomography (CBT, in black) and SLC-based backprojection (SLC-BP, in red).

While coherence-based tomography achieves a standard deviation of $\sigma \simeq 2.7 \mathrm{~m}$, SLC-based tomography gives a value of $\sigma \simeq 34.3 \mathrm{~m}$. The residual mismatch between the location of the selected phase center and the reference DEM is also due to the fact that some phase centers detected via tomography are, as tomograms in preceding sections have shown, located within the volume. This type of mismatch may be reduced further by refining the selection of the candidate phase centers, but this is out of the scope of the present analysis. In summary, the extracted DEM information is reliable and consistent with the TanDEM-X DEM. Coherencebased tomography can therefore also yield this kind of information.

\section{Tomographic Impulse Response Performance}

The evaluation of the tomographic imaging performance based on volume scatterers is generally still an open research topic due to the presence of multiple phase centers in height. Nevertheless, the results presented in this section aim to evaluate the performance in terms of impulse response analysis. Coherence-based 
tomography results are evaluated with respect to the other methods considered in the previous sections (SLCbased backprojection, Capon and Capon based on a filtered covariance matrix). A point target evaluation is performed under the assumption of a single layer scenario, since this corresponds to a vertical point scatterer. As in previous sections, the evaluation is based on the data acquired over the Brazilian state of Rondônia due to the presence of clear cuts within the site that can be considered as ground-truth. To perform the comparison, the TanDEM-X DEM height offset was removed from the tomograms, such that a peak response located around $0 \mathrm{~m}$ height is expected. Figure 12 shows the tomographic profiles retrieved along roughly $1 \mathrm{~km}$ of clear cuts for the different methods. This result consists of 500 superimposed retrievals (the azimuth sampling is about $2 \mathrm{~m}$ ) for all the methods considered. This type of evaluation allows both the accuracy as well as the stability of the retrieval to be assessed.

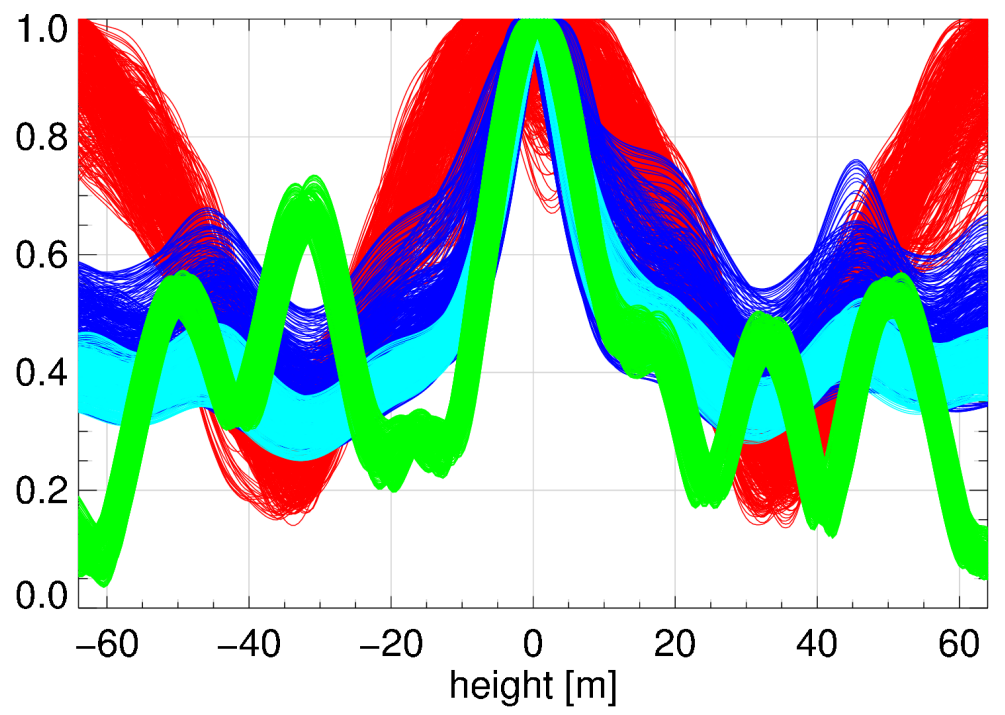

Figure 12: Superposition of 500 vertical profiles achieved with SLC-based backprojection (red), coherence-based tomography (green), Capon (blue), and Capon based on the filtered covariance matrix (cyan).

The results suggest that SLC-based backprojection (red) is the most unstable method due to its sensitivity to temporal changes. This weakness is partially compensated for the data-driven Capon approach (blue). The coherence-based inversion results in the most accurate and stable peak location and, on average, in a finer achieved resolution (green) compared to both alternative methods. As mentioned earlier, this is due to the fact that coherence-base tomography can take advantage of the quasi-simultaneous nature of the acquisitions. The Capon retrieval achieved after covariance matrix filtering (cyan) is the best result, in terms of average resolution and stability, among the SLC-based approaches. It is worth mentioning that the higher sidelobes at $\pm 30 \mathrm{~m}$ for the coherence-based implementation are due to the sub-optimal sampling of the interferometric baselines along the tomographic aperture. This state of affairs does not, however, mean that conclusions cannot be drawn concerning the performance of this approach within the relevant range of 
heights or, in general, with respect to future missions specifically designed for tomography.

\section{Conclusions}

The study evaluates the potential of coherence-based SAR tomography. A spaceborne implementation of tomography in which sensors acquire data in a quasi-simultaneous manner is considered. According to the Van Cittert-Zernike theorem, the impact of temporal decorrelation can be greatly reduced by coherently combining the set of single-pass acquisitions. The performance of this approach has been analyzed and compared to classical SLC-based techniques. In particular, the tomographic implementation considered is seen to perform as well as SLC super-resolution methods such as Capon, by exploiting the fact that the useful information is primarily contained in the set of single-pass coherences.

This outcome is relevant in view of the global effort to develope future systems featuring either twin or companion satellites to better control the temporal decorrelation issue and achieve the desired information retrieval capability. Thanks to the baseline diversity in the beginning of the TanDEM-X science phase and the flexibility of the radar systems involved in the mission, TanDEM-X represents a unique testbed for future spaceborne SAR missions. It is important to note that the results presented are not within the primary focus of applications for X-band data and that the TanDEM-X mission was never intended to suit tomography over vegetation. In addition, future TanDEM-X products, such as forest classification maps [Mart 18], stand to profit from the three-dimensional information attainable by means of SAR tomography for validation purposes.

To investigate possible application scenarios, stacks of data acquired over both boreal and tropical forest were analyzed. Despite the large acquisition time span of 2-3 months, SAR tomography could successfully be applied to retrieve vertical vegetation profiles. In addition, a new approach to mitigating the effects of temporal decorrelation via covariance matrix filtering was developed and was seen to produce encouraging results.

The analysis has been validated using the TanDEM-X DEM, thereby cross-validating the DEM itself for vegetated areas. Indeed, the DEM was seen to follow the top of the vegetation when compared to the tomographic retrievals. In addition, a coherence-based tomographic DEM was derived and showed a very good agreement with the TanDEM-X DEM. A seperate point target analysis was carried out and confirmed the expected advantages of coherence-based approach with respect to classical SLC-based techniques.

In conclusion, the three-dimensional resolution capabilities achieved by coherence-based tomography would allow the inner structure of volume scatterers, such as vegetation, to be directly measured on a global scale. In this context, interest in the estimation of the layers composing forests is driven by the fact that 
this structural parameter is of central importance in properly understanding the evolution of the biosphere [Cazc 17].

The results of the analyses presented, support the realization of dedicated spaceborne missions, in which the wavelength and the available baselines are specifically designed to optimally meet the requirements of tomography. In this context, missions like Tandem-L would, due to the penetration capabilities of L-band, provide groundbreaking measurements essential to breakthroughs in comprehending the Earth's ecosystem dynamics.

\section{References}

[Anno 15] "Announcement of opportunity: TanDEM-X science phase.". 2014-2015. https://tandemxscience.dlr.de/pdfs/TD-PD-PL_0032TanDEM-X_Science_Phase.pdf.

[Bohn 17] F. J. Bohn and A. Huth. "The importance of forest structure to biodiversity-productivity relationships". Royal Society open science, Vol. 4, No. 1, p. 160521, 2017.

[Cazc 17] V. Cazcarra-Bes, M. Tello-Alonso, R. Fischer, M. Heym, and K. Papathanassiou. "Monitoring of Forest Structure Dynamics by Means of L-Band SAR Tomography". Remote Sensing, Vol. 9, No. 12, p. 1229, 2017.

[Clou 06] S. R. Cloude. "Polarization coherence tomography". Radio Science, Vol. 41, No. 4, 2006.

[Davi 14] M. Davidson, N. Gebert, B. Carnicero Dominguez, F. Fois, and P. Silvestrin. "SAOCOM-CS: a passive companion mission to SAOCOM for single-pass L-band SAR bistatic interferometry and tomography". In: International Geoscience and Remote Sensing Symposium, pp. 1-4, 2014.

[Gini 02] F. Gini, F. Lombardini, and M. Montanari. "Layover solution in multibaseline SAR interferometry". IEEE Transactions on Aerospace and Electronic Systems, Vol. 38, No. 4, pp. 1344-1356, 2002.

[Krie 07] G. Krieger, A. Moreira, H. Fiedler, I. Hajnsek, M. Werner, M. Younis, and M. Zink. "TanDEMX: A Satellite Formation for High-Resolution SAR Interferometry". IEEE Transactions on Geoscience and Remote Sensing, Vol. 45, No. 11, pp. 3317-3341, Nov. 2007.

[Krie 09] G. Krieger, I. Hajnsek, K. Papathanassiou, M. Eineder, M. Younis, F. D. Zan, P. Prats, S. Huber, M. Werner, H. Fiedler, et al. "The Tandem-L mission proposal: Monitoring Earth's dynamics with high resolution SAR interferometry". In: 2009 IEEE Radar Conference, pp. 1-6, 2009. 
[Mart 18] M. Martone, P. Rizzoli, C. Wecklich, C. González, J.-L. Bueso-Bello, P. Valdo, D. Schulze, M. Zink, G. Krieger, and A. Moreira. "The global forest/non-forest map from TanDEM-X interferometric SAR data". Remote Sensing of Environment, Vol. 205, pp. 352-373, 2018.

[More 15] A. Moreira, G. Krieger, I. Hajnsek, K. Papathanassiou, M. Younis, P. Lopez-Dekker, S. Huber, M. Villano, M. Pardini, M. Eineder, et al. "Tandem-L: A Highly Innovative Bistatic SAR Mission for Global Observation of Dynamic Processes on the Earth's Surface". IEEE Geoscience and Remote Sensing Magazine, Vol. 3, No. 2, pp. 8-23, 2015.

[Nann 09] M. Nannini, R. Scheiber, and A. Moreira. "Estimation of the minimum number of tracks for SAR tomography". IEEE Transactions on Geoscience and Remote Sensing, Vol. 47, No. 2, pp. 531-543, 2009.

[Nann 12] M. Nannini, R. Scheiber, R. Horn, and A. Moreira. "First 3-D reconstructions of targets hidden beneath foliage by means of polarimetric SAR tomography". IEEE Geoscience and Remote Sensing Letters, Vol. 9, No. 1, pp. 60-64, 2012.

[Nann 16] M. Nannini, P. Prats-Iraola, R. Scheiber, N. Yague-Martinez, F. Minati, F. Vecchioli, M. Costantini, S. Borgstrom, P. De Martino, V. Siniscalchi, et al. "Sentinel-1 mission: results of the InSARap project". In: Proceedings of EUSAR 2016, pp. 1-4, 2016.

[Nann 17] M. Nannini, M. Martone, P. Rizzoli, P. Prats-Iraola, M. Rodriguez-Cassola, and A. Moreira. "Spaceborne demonstration of coherent based SAR tomography for future companion satellite SAR missions". In: IEEE International Geoscience and Remote Sensing Symposium, July 2017.

[NASA 00] NASA. "Biome study". https://earthobservatory.nasa.gov/Experiments/Biome/, 2000.

[Onli 16] W. W. Online. "Precipitation". https://www.worldweatheronline.com/ rondonia-weather-averages/rondonia/br .aspx, 2016.

[Pard 13] M. Pardini, A. Torano-Caicoya, F. Kugler, and K. Papathanassiou. "Estimating and understanding vertical structure of forests from multibaseline TanDEM-X Pol-InSAR data". In: Geoscience and Remote Sensing Symposium (IGARSS), 2013 IEEE International, pp. 4344-4347, IEEE, 2013.

[Prat 10] P. Prats, M. Rodriguez-Cassola, L. Marotti, M. Naninni, S. Wollstadt, D. Schulze, N. TousRamon, M. Younis, G. Krieger, and A. Reigber. "TAXI: A versatile processing chain for experimental TanDEM-X product evaluation". In: International Geoscience and Remote Sensing Symposium, pp. 4059-4062, July 2010. 
[Reig 00] A. Reigber and A. Moreira. "First Demonstration of Airborne SAR Tomography Using Multibaseline L-Band Data". IEEE Transactions on Geoscience and Remote Sensing, Vol. 38, No. 5, pp. 2142-2152, Sep. 2000.

[Sima 12] M. Simard, S. Hensley, M. Lavalle, R. Dubayah, N. Pinto, and M. Hofton. "An empirical assessment of temporal decorrelation using the uninhabited aerial vehicle synthetic aperture radar over forested landscapes". Remote Sensing, Vol. 4, No. 4, pp. 975-986, 2012.

[Teba 08] S. Tebaldini. "Forest SAR tomography: a covariance matching approach". In: 2008 IEEE Radar Conference, pp. 1-6, 2008.

[Teba 16] S. Tebaldini, T. Nagler, H. Rott, and A. Heilig. "Imaging the internal structure of an alpine glacier via L-band airborne SAR tomography". IEEE Transactions on Geoscience and Remote Sensing, Vol. 54, No. 12, pp. 7197-7209, 2016.

[Teba 17] S. Tebaldini and L. Ferro-Famil. "SAR tomography from bistatic single-pass interferometers". In: IEEE International Geoscience and Remote Sensing Symposium, July 2017.

[Zebk 92] H. A. Zebker and J. Villasenor. "Decorrelation in interferometric radar echoes". IEEE Transactions on Geoscience and Remote Sensing, Vol. 30, No. 5, pp. 950-959, 1992.

[Zink 14] M. Zink, M. Bachmann, B. Brautigam, T. Fritz, I. Hajnsek, A. Moreira, B. Wessel, and G. Krieger. "TanDEM-X: the new global DEM takes shape". IEEE Geoscience and Remote Sensing Magazine, Vol. 2, No. 2, pp. 8-23, 2014. 